\title{
TOWARDS AN ONTOLOGY-BASED MODEL FOR USER PROFILE REPRESENTATION IN A CLOUD INFRASTRUCTURE
}

\author{
Vassilis Koutkias \\ Center for Research and Technology Hellas \\ 6th Km Charilaou-Thermi Rd, P.O. BOX 361, 57001, Thessaloniki, Greece \\ vkoutkias@certh.gr \\ Evangelos Bekiaris \\ Center for Research and Technology Hellas \\ 6th Km Charilaou-Thermi Rd, P.O.BOX 361, 57001, Thessaloniki, Greece \\ abek@certh.gr \\ Nicos Maglaveras \\ Center for Research and Technology Hellas \\ 6th Km Charilaou-Thermi Rd, P.O. BOX 361, 57001, Thessaloniki, Greece \\ nicmag@certh.gr \\ Maria Gkemou \\ Center for Research and Technology Hellas \\ 6th Km Charilaou-Thermi Rd, P.O.BOX 361, 57001, Thessaloniki, Greece \\ mgemou@certh.gr
}

\begin{abstract}
This paper presents an approach for the semantic representation of user profiles through an ontology conducted in the scope of the Cloud4all project. Employing the cloud computing paradigm, Cloud4allaims torealize a global public inclusive infrastructure that will reinforce accessibility of information and communication technologies(ICT). The proposed ontology aims to provide the required representation formalism to sufficiently express personal user needs and preferences based on domain knowledge and the association of interaction-related concepts in order to support automatic reasoning mechanisms for user profile management.
\end{abstract}

Keywords: Cloud Computing, Ontologies, User Needs and Preferences, Accessible ICT 


\section{INTRODUCTION}

While information and communication technologies (ICT) are becoming more sophisticated, adaptable, and personalized, there are still major obstacles towards achieving accessible ICT for all people according to their specific needs and preferences. Addressing this goal remains an important research and development challenge. Cloud4all is an EU-founded international project (http://cloud4all.info/) aiming to address this challenge through the realization of a global public inclusive infrastructure (http://gpii.net/). In this regard, Cloud4all develops the necessary models and tools by deploying a cloud computing infrastructure.This paper presents the part of Cloud4all that concerns the semantic representation of user profiles, conceived as sets of user needs and preferences. The semantic approach relies on an ontology model, aiming to provide the required representation formalism to sufficiently express personal user needs and preferences in order to support automatic reasoning mechanisms for user profile management. In the following sections, we present the major design and development principles of our work as well as the main ontology concepts and structure.

\section{THE PROPOSED ONTOLOGY}

\subsection{Rationale, Design and Development Considerations}

The proposed ontology aims to introduce a semantic model that will (a) offer the required expressiveness for describing user profiles based on personal needs and preferences across applications, platforms, and devices; (b) provide the basis for developing tools to facilitate user profile initialization and management; and (c) provide the foundation for developing semantics-based matchmaking approaches among user needs and preferences and applications/services. The elaborated approach takes into account standards devoted to user needs and preferences modeling, e.g., ISO/IEC $24751^{1}$ and ETSI TS $202746^{2}$, as well as new standardization efforts aiming to reinforce its exploitation potential and generalization. In particular, a new standardization effort aiming to propose an updated version of ISO/IEC 24751(http://wiki.fluidproject.org/display/ISO24751/AccessForAll+Workin g+Group) conducted by the AccessForAll working group is influential to this work. Thus, the ontology employs the proposedregistry of common terms as its core partby using itstermsto express generic needs and preferences, independent of ICT solutions.

The content of the registry is exploited via the ontology by introducing classification schemes such as interaction channels (e.g. VisualInputInteractionChannel) and interface elements (e.g. a Window). 
The above groupings enable us to express high-level interaction requirements in terms of registry items; e.g., a user's need for blocking the VisualInputInteractionChannel (e.g. while driving) shall be associated with a subset of registry items which are rather low-level. This is particularly important for tasks such as profile initialization, in which the user cannot be asked about all possible needs and preferences he/she might have. In such case, the user could be asked to provide basic (high-level) information that will be mapped to low-level configuration settings for adapting ICT. The ontology development relies on an iterative and incremental approach ${ }^{3}$ aiming to support profile initialization and management as well as semantic matchmaking between user needs/preferences and available applications/services. The ontologyis encoded in the Web Ontology Language (OWL) and developed in Protégé (http://protege.stanford.edu/).

\subsection{Ontology Structure and Main Concepts}

The first-level hierarchy of the proposed ontology involves the following major concepts, which may be grouped according to their semantics as follows:

a) Core-concepts, comprising of the following classes:

- RegistryProperty: Instances of this class correspond to entries defined in the AccessForAllRegistry (the class structure is marked in red in Figure 1).

- UserPreference: A specific UserPreference that is associated with one or more instances of the RegistryProperty class.

- Condition: A Condition according to which a specific UserPreference is applicable, e.g. "Morning", "AtHome", "WhileDriving", etc. The range of this class is quite wide and will be determined in a later version of the ontology.

- UserProfile: A specific user's profile defined as a group of specific UserPreference instances.

- User: A user of the Cloud4All platform for whom a UserProfileis defined.

The relation among the above Core-concepts is illustrated in Figure 2.

b) Context-related concepts, corresponding to the following classes:

- Application: The application(s)to which the UserProfile shall be applied, e.g. a "Web browser", a "ScreenReader", a"Text Editor", etc.

- Device: The device(s)to which the UserProfile shall be applied, e.g. a "Smartphone", a "DTV", a "Desktop Computer", etc.

- OperatingSystem: The OSsto which the UserProfile shall be applied. 
- Setting: A specific configuration setting that a Device, OperatingSystem or Application may offer, also expressed as an instance of the RegistryProperty.

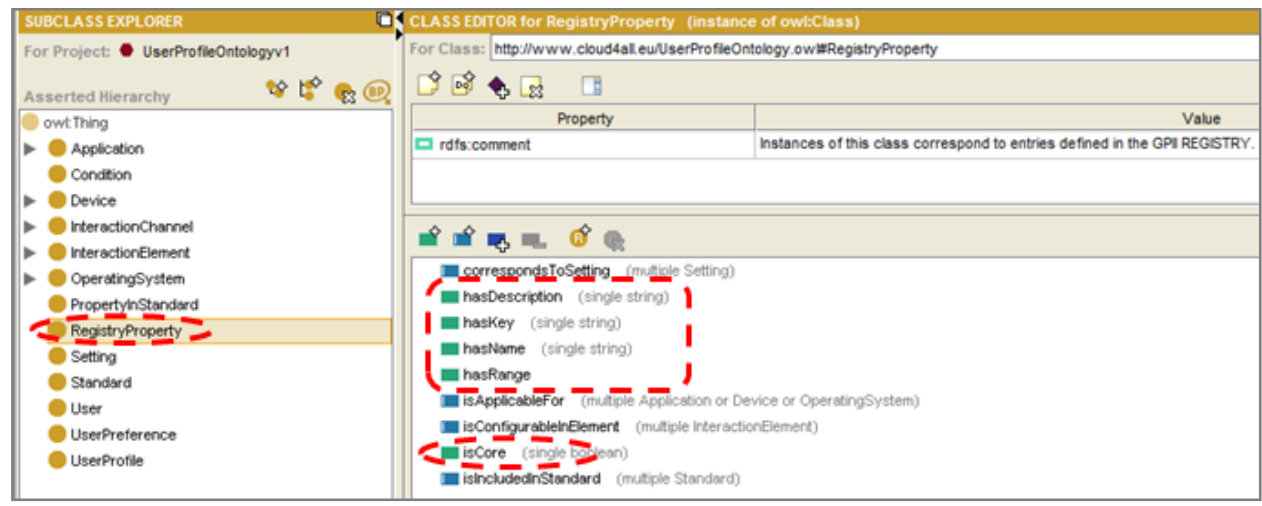

Figure 1. First-level ontology hierarchy highlighting the definition of the RegistryProperty class as depicted in Protégé

c) Conceptual views related concepts, expressed through classes such as:

- InteractionChannel: Involves the various user interaction channels that are available through ICT. Two subclasses are defined, namely,InputInteractionChannel and OutputInteractionChannel, along with consecutive subclasses. This class aims to cluster instances of the RegistryProperty class according to user interaction channels.

- InteractionElement: Involves the various interaction elements/aspects that are configurable, e.g. "Layout","Fonts","Window” etc., per Application/OperatingSystem.

d) Standards-related concepts, expressed through the following classes:

- PropertyInStandard: Corresponds to a set of UserPreference instances that are defined/included in relevant standards.

- Standards: Refers to standards relevant to user profiling, e.g. ISO/IEC 27451.

\section{CONCLUSION}

The current paper presents work in progress towards the development of a semantic model for effectively expressing user profiles ofpersonal needs and preferences, aiming to reinforce accessible ICT. The proposed ontology defines core user profile concepts, such as user preferences, user profile, user, etc. and associates them with context-related concepts as well as user interaction elements. The ontology is currently being populated with a large number of instances. Furthermore, its evaluation concerning its 
expressiveness and applicability is under design through various use case scenarios coping with diverse, actual user needs and preferences ${ }^{4}$. Further information concerning this work is available at http://wiki.gpii.net/index.php/Ontologies.

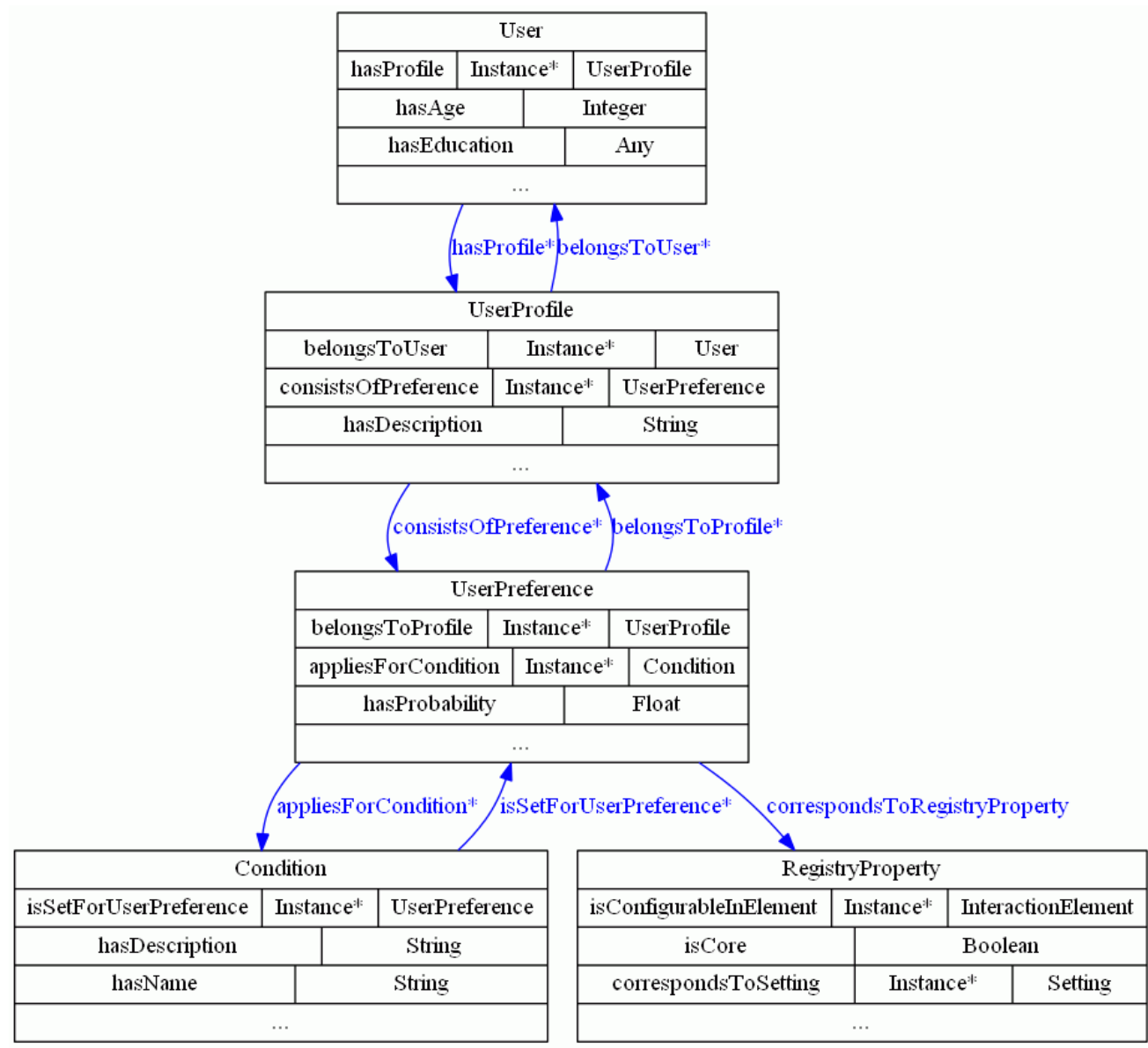

Figure 2. Association among the Core-concepts (User, UserProfile, UserPreference, Condition and RegistryProperty).

\section{ACKNOWLEDGMENT}

The research leading to these results has received funding from the European Community's Seventh Framework Program (FP7/2007-2013) under Grant Agreement $\mathrm{n}^{\circ} 289016$ - the Cloud4all project. The authors would like to thank the Cloud4all partners for their influential contributions in the current work.

\section{REFERENCES}

[1] ISO/IEC 24751, Information technology — Individualized adaptability 
and accessibility in e-learning, education and training, 2008.

[2] ETSI ES 202 746, Human Factors (HF); Personalization and User Profile Management; User Profile Preferences and Information, 2010.

[3] T.R. Gruber, Toward principles for the design of ontologies used for knowledge sharing. International Journal Human-Computer Studies, 43(5-6), p907-928, 1995. http://dx.doi.org/10.1006/ijhc.1995.1081.

[4] L. Hella, and J. Krogstie, A structured evaluation to assess the reusability of models of user profiles. LNBIP, 50, p220-233, 2010. http://dx.doi.org/10.1007/978-3-642-13051-9_19. 